H. UEDA

KODAI MATH. J.

5 (1982). 89-99

\title{
TWO RESULTS ASSOCIATED WITH SPREAD RELATION
}

\author{
By HidehaRu UEdA
}

\section{Introduction.}

Let $u=u_{1}-u_{2}$ be nonconstant, where $u_{1}$ and $u_{2}$ are subharmonic in the plane. For such a function $u$, we will write

$$
N(r, u)=\frac{1}{2 \pi} \int_{0}^{2 \pi} u\left(r e^{2 \theta}\right) d \theta
$$

Then the Nevanlinna characteristic of $u$ is defined by

$$
T(r) \equiv T(r, u)=N\left(r, u^{+}\right)+N\left(r, u_{2}\right) .
$$

With this $T(r, u)$, the order and lower order of $u$ are defined as follows:

$$
\begin{aligned}
& \rho=\varlimsup_{r \rightarrow \infty} \frac{\log T(r, u)}{\log r} \quad \text { (the order of } u \text { ), } \\
& \mu=\varliminf_{r \rightarrow \infty} \frac{\log T(r, u)}{\log r} \quad \text { (the lower order of } u \text { ). }
\end{aligned}
$$

Further, we use the notation

$$
\delta(\infty) \equiv \delta(\infty, u)=1-\varlimsup_{r \rightarrow \infty} \frac{N\left(r, u_{2}\right)}{T(r, u)} .
$$

(Throughout this paper, $|E|$ denotes the one-dimensional Lebesgue measure of the set $E$.)

Assume that $\mu<\infty$. Then, Baernstein [3] proved the following inequality.

Spread Relation: For any fixed $b \in(-\infty, \infty)$,

$$
\varlimsup_{r \rightarrow \infty}\left|\left\{\theta: u\left(r e^{\imath \theta}\right)>b\right\}\right| \geqq \min \left\{\frac{4}{\mu} \sin ^{-1}\left(\frac{\delta(\infty)}{2}\right)^{1 / 2}, 2 \pi\right\} .
$$

In connection with this relation, we show the following result.

THEOREM 1. Let $u=u_{1}-u_{2}$ be nonconstant, where $u_{1}$ and $u_{2}$ are subharmonic in the plane. Suppose $\mu<\infty$ and $\delta(\infty)>0$. Let $\lambda$ be a number satısfying

$$
\lambda>\mu, \quad \frac{4}{\lambda} \sin ^{-1}\left(\frac{\delta(\infty)}{2}\right)^{1 / 2} \leqq 2 \pi .
$$

Received September 18, 1980. 
Then, for any fixed $b \in(-\infty, \infty)$,

$$
\overline{\log \operatorname{dens}}\left\{r:\left|\left\{\theta: u\left(r e^{2 \theta}\right)>b\right\}\right| \geqq \frac{4}{\lambda} \sin ^{-1}\left(\frac{\delta(\infty)}{2}\right)^{1 / 2}\right\} \geqq 1-\frac{\mu}{\lambda} .
$$

In order to show Theorem 1 , it is sufficient to prove the following

THEOREM 2. Let $u=u_{1}-u_{2}$ be nonconstant, where $u_{1}$ and $u_{2}$ are subharmonic in the plane. Suppose $\mu<\infty$. Let $\delta$ and $\lambda$ be numbers satisfying

$$
\lambda>\mu, \quad 0<\delta \leqq 1, \quad \frac{4}{\lambda} \sin ^{-1}\left(\frac{\delta}{2}\right)^{1 / 2} \leqq 2 \pi .
$$

Further, suppose that there exists a $r_{0}>0$ such that $r \geqq r_{0}$ implies

$$
N\left(r, u_{2}\right) \leqq(1-\delta) T(r, u)+O(1) .
$$

Then, for any fixed $b \in(-\infty, \infty)$,

$$
\overline{\log \operatorname{dens}}\left\{r:\left|\left\{\theta: u\left(r e^{2 \theta}\right)>b\right\}\right| \geqq \frac{4}{\lambda} \sin ^{-1}\left(\frac{\delta}{2}\right)^{1 / 2}\right\} \geqq 1-\frac{\mu}{\lambda} .
$$

If we assume that $\rho<\infty$, we have the following

THEOREM 3. Let $u=u_{1}-u_{2}$ be nonconstant, where $u_{1}$ and $u_{2}$ are subharmonic in the plane. Suppose $\rho<\infty$ and $\delta(\infty)>0$. Let $\lambda$ be a number satisfying

$$
\lambda>\rho, \frac{4}{\lambda} \sin ^{-1}\left(\frac{\delta(\infty)}{2}\right)^{1 / 2} \leqq 2 \pi .
$$

Then, for any fixed $b \in(-\infty, \infty)$,

$$
\underline{\log \operatorname{dens}}\left\{r:\left|\left\{\theta: u\left(r e^{\imath \theta}\right)>b\right\}\right| \geqq \frac{4}{\lambda} \sin ^{-1}\left(\frac{\delta(\infty)}{2}\right)^{1 / 2}\right\} \geqq 1-\frac{\rho}{\lambda} .
$$

Again, we prove the following Theorem 4 in place of Theorem 3.

THEOREM 4. Let $u=u_{1}-u_{2}$ be nonconstant, where $u_{1}$ and $u_{2}$ are subharmonic in the plane. Suppose $\rho<\infty$. Let $\delta$ and $\lambda$ be numbers satisfying

$$
\lambda>\rho, \quad 0<\delta \leqq 1, \quad \frac{4}{\lambda} \sin ^{-1}\left(\frac{\delta}{2}\right)^{1 / 2} \leqq 2 \pi .
$$

Further, suppose that there exists a $r_{0}>0$ such that $r \geqq r_{0}$ implies

$$
N\left(r, u_{2}\right) \leqq(1-\delta) T(r, u)+O(1) .
$$

Then, for any fixed $b \in(-\infty, \infty)$,

$$
\underline{\log \operatorname{dens}}\left\{r:\left|\left\{\theta: u\left(r e^{i \theta}\right)>b\right\}\right| \geqq \frac{4}{\lambda} \sin ^{-1}\left(\frac{\delta}{2}\right)^{1 / 2}\right\} \geqq 1-\frac{\rho}{\lambda} .
$$

Theorems 1 and 3 may be regarded as analogues of Barry's results [5], [4] on the $\cos \pi \rho$ theorem. The proof of Theorems 2 and 4 is accomplished by 
combining Barry's method with Baernstein's "star function" method.

For $u=u_{1}-u_{2}$, we define

$$
u^{*}\left(r e^{\imath \theta}\right)=\sup _{E} \frac{1}{2 \pi} \int_{E} u\left(r e^{\imath \omega}\right) d \omega \quad(r>0,0 \leqq \theta \leqq \pi),
$$

where the sup is taken over all sets $E \subset[-\pi, \pi]$ with $|E|=2 \theta$, and define

$$
u \#\left(r e^{\imath \theta}\right)=u^{*}\left(r e^{\imath \theta}\right)+N\left(r, u_{2}\right) \text {. }
$$

In [2], Baernstein proved that $u^{\#}(z)$ is subharmonic in the upper half plane.

Note that we may prove Theorems 2 and 4 under the following additional conditions.

(i) $u_{1}$ and $u_{2}$ are harmonic in a neighborhood of 0 .

(ii) $b=0$.

(iii) $u_{1}(z) \geqq u_{2}(z)$ for all $z, u_{1}(0)=u_{2}(0)=0$.

For the details, see [3, p. 89].

\section{Preliminaries for the proof of Theorem 2 .}

1.1. A function $h(z)$. Set $\beta$ and $\gamma$ as follows:

$$
\beta=\frac{2}{\lambda} \sin ^{-1}\left(\frac{\delta}{2}\right)^{1 / 2}, \quad \gamma=\frac{\beta}{\pi} .
$$

Then

$$
\alpha \equiv \gamma \lambda=\frac{2}{\pi} \sin ^{-1}\left(\frac{\delta}{2}\right)^{1 / 2} \leqq 1 / 2 .
$$

Fix $R>0$ and define

$$
B(t)=\left\{\begin{array}{l}
T\left(t^{\gamma}\right) \quad(0 \leqq t \leqq R), \\
T_{1}\left(R^{\gamma}-\right) \log \frac{t}{R}+T\left(R^{\gamma}\right) \quad(R \leqq t<\infty),
\end{array}\right.
$$

where $T_{1}\left(t^{r}\right)$ denotes the logarithmic derivative of the function $t \rightarrow T\left(t^{r}\right)$. Then $B(t)$ is a convex nondecreasing function of $\log t$, and the Poisson integral

$$
h(z)=\frac{1}{\pi} \int_{0}^{\infty} \frac{r \sin \theta}{t^{2}+r^{2}+2 t r \cos \theta} B(t) d t \quad\left(z=r e^{2 \theta}\right)
$$

is harmonic in the slit plane $|\arg z|<\pi$, is zero on the positive axis and tends to $B(r)$ as $\theta \rightarrow \pi-$. Further,

$$
h_{\theta}\left(r e^{\imath \theta}\right)=\frac{1}{\pi} \int_{0}^{\infty} \log \left|1+\frac{r}{t} e^{\imath \theta}\right| d B_{1}(t) \quad(|\theta|<\pi)
$$

and 


$$
\lim _{\theta \rightarrow \pi-} \frac{B(r)-h\left(r e^{2 \theta}\right)}{\pi-\theta}=\lim _{\theta \rightarrow \pi-} h_{\theta}\left(r e^{2 \theta}\right)=\frac{1}{\pi} \int_{0}^{\infty} \log \left|1-\frac{r}{t}\right| d B_{1}(t)
$$

hold, where $B_{1}(t)$ is the logarithmic derivative of the logarithmically convex nondecreasing function $B(t)$, which were established in $\S 3$ of [1].

Since $u(z)$ is nonconstant (cf. $\left[3\right.$, p. 83, b)]), there exists a number $r_{2}>0$ such that $B_{1}\left(r_{2}\right)>0$, provided that $R\left(>2 r_{2}\right)$ is large enough.

By (1.2),

$$
\begin{aligned}
& \pi h_{\theta}\left(\frac{R}{2}\right)=\int_{0}^{R} \log \left(1+\frac{R}{2 t}\right) d B_{1}(t) \\
&=B_{1}(R-) \log \frac{3}{2}+B(R) \frac{1}{3}+\int_{0}^{R} \frac{R / 2}{(t+R / 2)^{2}} B(t) d t \\
& \leqq B_{1}(R-) \frac{3}{2}+B(R) \leqq \int_{R}^{R e} \frac{B_{1}(t)}{t} d t+B(R) \\
& \leqq B(R e)+B(R) \leqq 2 B(R e)=2\left\{T_{1}\left(R^{\gamma}-\right)+T\left(R^{\gamma}\right)\right\} \\
& \leqq 2\left\{\int_{R}^{R e} \frac{T_{1}\left(t^{\gamma}\right)}{t} d t+T\left(R^{\gamma}\right)\right\} \leqq 2\left\{T\left(R^{\gamma} e^{\gamma}\right)+T\left(R^{\gamma}\right)\right\} \leqq 4 T\left(R^{\gamma} e^{\gamma}\right), \quad \text { i. e. } \\
& h_{\theta}\left(\frac{R}{2}\right) \leqq \frac{4}{\pi} T\left(R^{\gamma} e^{\gamma}\right)
\end{aligned}
$$

Also,

$$
\begin{aligned}
\pi h_{\theta}\left(r_{2}\right) & =\int_{0}^{R} \log \left(1+\frac{r_{2}}{t}\right) d B_{1}(t) \geqq \int_{0}^{r_{2}} \log \left(1+\frac{r_{2}}{t}\right) d B_{1}(t) \\
& =B_{1}\left(r_{2}\right) \log 2+\int_{0}^{r_{2}} B_{1}(t) \frac{r_{2}}{r_{2}+t} \frac{d t}{t} \geqq B_{1}\left(r_{2}\right) \log 2>0 .
\end{aligned}
$$

1.2. A function $h_{1}(z)$. Let $D$ be the disk $\{z:|z|<R\}$ and let $h_{1}(z)$ be the bounded harmonic function in $D$ defined by

$$
\begin{aligned}
h_{1}\left(r e^{2 \theta}\right)= & \frac{1}{2 \pi} \int_{0}^{\pi} T\left(R^{r}\right) \frac{R^{2}-r^{2}}{R^{2}+r^{2}-2 R r \cos (\theta-t)} d t \\
& +\frac{1}{2 \pi} \int_{\pi}^{2 \pi}\left(-T\left(R^{r}\right)\right) \frac{R^{2}-r^{2}}{R^{2}+r^{2}-2 R r \cos (\theta-t)} d t .
\end{aligned}
$$

Then it is easy to see that

$$
h_{1}\left(r e^{2 \theta}\right) \begin{cases}>0 & (0<\theta<\pi), \\ =0 & (\theta=0, \pi), \\ <0 & (\pi<\theta<2 \pi) .\end{cases}
$$

By Proposition 6 of [1],

$$
\left|\left(h_{1}\right)_{\theta}(z)\right| \leqq A \frac{|z|}{R} T\left(R^{r}\right) \quad(|z| \leqq R / 2),
$$


where $A(>0)$ is an absolute constant.

From (1.6) we deduce that

$$
\left(h_{1}\right)_{\theta}(-t) \leqq 0 \quad(0<t<R) .
$$

1.3. A function $F(t)$. By (1.1), $\alpha \leqq 1 / 2$. Then, for $0<R_{1}<R_{2}$,

$$
\begin{gathered}
\int_{R_{1}}^{R_{2}}\left\{\log \left|1-\frac{t}{s}\right|-\cos \pi \alpha \cdot \log \left(1+\frac{t}{s}\right)\right\} \frac{d t}{t^{1+\alpha}} \\
\quad>C(\alpha) \frac{\log \left(1+R_{1} / s\right)}{R_{1}{ }^{\alpha}}-K(\alpha) \frac{\log \left(1+R_{2} / s\right)}{R_{2}{ }^{\alpha}},
\end{gathered}
$$

where $C(\alpha)=(1-\cos \pi \alpha) / \alpha$ and $K(\alpha)$ is some number greater than $C(\alpha)$ (cf. [7, (21), (22)]). We take the integral $d B_{1}(s)$ of each term in (1.9). Using (1.2) and (1.3), we have

$$
\int_{R_{1}}^{R_{2}}\left\{h_{\theta}(-t)-\cos \pi \alpha h_{\theta}(t)\right\} \frac{d t}{t^{1+\alpha}}>C(\alpha) \frac{h_{\theta}\left(R_{1}\right)}{R_{1}{ }^{\alpha}}-K(\alpha) \frac{h_{\theta}\left(R_{2}\right)}{R_{2}{ }^{\alpha}} .
$$

so that

$$
\int_{R_{1}}^{R_{2}}\left\{h_{\theta}(-t)-h_{\theta}(t)+C(\alpha) t \frac{\partial}{\partial t} h_{\theta}(t)\right\} \frac{d t}{t^{1+\alpha}}+(K(\alpha)-C(\alpha)) \frac{h_{\theta}\left(R_{2}\right)}{R_{2}{ }^{\alpha}}>0 .
$$

Here we put

$$
F(t)=h_{\theta}(-t)+\left(h_{1}\right)_{\theta}(-t)-h_{\theta}(t)+C(\alpha) t-\frac{\partial}{\partial t} h_{\theta}(t) .
$$

Then it follows from (1.4), (1.7) and (1.10) that, for $0<r \leqq R / 2$,

$$
\begin{aligned}
\int_{r}^{R / 2} F(t) \frac{d t}{t^{1+\alpha}}> & -(K(\alpha)-C(\alpha)) \frac{(4 / \pi) T\left(R^{\gamma} e^{\gamma}\right)}{(R / 2)^{\alpha}}-A \frac{T\left(R^{r}\right)}{R} \int_{r}^{R / 2} \frac{d t}{t^{\alpha}} \\
& >-\left\{(K(\alpha)-C(\alpha)) \frac{2^{2+\alpha}}{\pi}+-\frac{A}{(1-\alpha) 2^{1-\alpha}}\right\} \frac{T\left(R^{\gamma} e^{r}\right)}{R^{\alpha}} \\
& \equiv-K_{1}(\alpha) \frac{T\left(R^{\gamma} e^{\gamma}\right)}{R^{\alpha}} .
\end{aligned}
$$

1.4. A function $H(z)$. Consider the harmonic function $H(z)$ in $D^{+}=\{z: z \in D$, $0<\arg z<\pi\}$ defined by

$$
H\left(r e^{\imath \theta}\right)=h\left(r e^{\imath \theta}\right)+\cos \pi \alpha h\left(r e^{\imath(\pi-\theta)}\right)+h_{1}\left(r e^{\imath \theta}\right)+A_{1}(\pi-\theta),
$$

where $A_{1}$ is a large positive constant independent of $R$. The boundary values of $H$ satisfy

$$
\begin{aligned}
& H(-r)=T\left(r^{r}\right) \quad(0 \leqq r<R), \\
& H(r)=\cos \pi \alpha T\left(r^{r}\right)+A_{1} \pi \quad(0<r<R), \\
& H\left(R e^{2 \theta}\right) \geqq T\left(R^{r}\right) \quad(0<\theta<\pi) .
\end{aligned}
$$


Now, set

Then by (1.12) we have

$$
v(z)=u^{\#}\left(z^{\gamma}\right)
$$

$$
\begin{aligned}
& v(-r)=u^{\#}\left(r^{r} e^{2 \beta}\right) \leqq T\left(r^{\gamma}\right)=H(-r) \quad(0 \leqq r<R), \\
& v(r)=u^{\#}\left(r^{r}\right)=N\left(r^{\gamma}, u_{2}\right) \leqq(1-\delta) T\left(r^{r}\right)+C=\cos \pi \alpha T\left(r^{\gamma}\right)+C \\
& \left(r \geqq r_{1} \equiv r_{0}^{1 / r}, C: \text { a positive constant }\right), \\
& v\left(R e^{2 \theta}\right)=u^{\#}\left(R^{\gamma} e^{i \gamma \theta}\right) \leqq T\left(R^{r}\right)<H\left(R e^{i \theta}\right) \quad(0<\theta<\pi) .
\end{aligned}
$$

Hence, if we choose $A_{1} \pi=\max \left\{C, N\left(r_{0}, u_{2}\right)\right\}$, then

$$
v(z) \leqq H(z) \quad\left(z \in D^{+}\right) .
$$

\section{Proof of Theorem 2.}

We define, for $0<r \leqq R / 2$,

$$
H_{1}(r)=K_{1}(\alpha) \frac{T\left(R^{\gamma} e^{\gamma}\right)}{R^{\alpha}} r^{\alpha}+r^{\alpha} \int_{0}^{R / 2} F(t) \frac{d t}{t^{1+\alpha}} .
$$

By (1.11), $H_{1}(r)>0$ for $0<r \leqq R / 2$. Set

$$
G_{1}(r)=H_{1}(r)+C(\alpha) h_{\theta}(r) .
$$

Then (2.1) implies

$$
r G_{1}^{\prime}(r)=\alpha H_{1}(r)+h_{\theta}(r)-h_{\theta}(-r)-\left(h_{1}\right)_{\theta}(-r)
$$

a. e. in $0<r \leqq R / 2$. By (1.2), $h_{\theta}(r)-h_{\theta}(-r) \geqq 0$ for $0<r \leqq R / 2$, and by (1.8), $-\left(h_{1}\right)_{\theta}(-r) \geqq 0$ for $0<r \leqq R / 2$. Hence, (2.3) shows that

$$
r G_{1}^{\prime}(r)>0 \quad \text { a.e. in } 0<r \leqq R / 2 .
$$

Since $G_{1}(r)$ is absolutely continuous in $r$ and its derivative is positive a. e., we know that $G_{1}(r)$ increases with $r$, and hence $\log G_{1}(r)$ is absolutely continuous. By (1.5) and (2.2),

$$
G_{1}\left(r_{2}\right)>C(\alpha) h_{\theta}\left(r_{2}\right) \geqq C(\alpha) B_{1}\left(r_{2}\right) \frac{\log 2}{\pi}>0 .
$$

Thus, we deduce from (2.1), (2.2), (1.4) and (2.5) that

$$
\begin{aligned}
\int_{r_{2}}^{R / 2} \frac{G_{1}^{\prime}(t)}{G_{1}(t)} & d t=\log G_{1}\left(\frac{R}{2}\right)-\log G_{1}\left(r_{2}\right) \\
& <\log \left\{K_{1}(\alpha) \frac{T\left(R^{\gamma} e^{\gamma}\right)}{2^{\alpha}}+C(\alpha) \frac{4}{\pi} T\left(R^{\gamma} e^{\gamma}\right)\right\}-\log \left\{C(\alpha) B_{1}\left(r_{2}\right) \frac{\log 2}{\pi}\right\} \\
& \equiv C_{1}+\log T\left(R^{\gamma} e^{\gamma}\right)
\end{aligned}
$$


where $C_{1}$ is a constant depending only on $\alpha, r_{2}$ and $u(z)$.

Let

and

$$
I=\left\{r:\left|\left\{\theta: u\left(r e^{i \theta}\right)>0\right\}\right|<2 \beta\right\}
$$

$$
J=\left\{r: r^{r} \in I\right\} \text {. }
$$

Suppose that $r \in I$ and put

$$
E(r)=\left\{\theta: u\left(r e^{i \theta}\right)>0\right\} .
$$

Then, since $u(z) \geqq 0$ everywhere and $|E(r)|<2 \beta$, we have

so that

$$
u^{\#}\left(r e^{i \beta}\right) \geqq N\left(r, u^{+}\right)+N\left(r, u_{2}\right)=T(r),
$$

Hence

$$
u^{\#}\left(r e^{i \beta}\right)=T(r) \quad(r \in I) .
$$

$$
v(-r)=u^{\#}\left(r^{r} e^{i \beta}\right)=T\left(r^{r}\right)=H(-r) \quad(r \in J \cap(0, R)) .
$$

It follows from (1.13) and (2.7) that, for $r \in J \cap(0, R)$,

$$
H_{\theta}(-r)=\lim _{\theta \rightarrow \pi-} \frac{H(-r)-H\left(r e^{i \theta}\right)}{\pi-\theta} \leqq v_{\theta}(-r)=\gamma u_{\theta}^{\#}\left(r^{\gamma} e^{2 \beta}\right) .
$$

(Existence of the limit follows from (1.3).) Let $\tilde{u}\left(r e^{i \theta}\right)$ denote the symmetric decreasing rearrangement of $u\left(r e^{i \theta}\right)$ (cf. [2, §3]). Then

$$
u_{\theta}^{\#}\left(r^{\gamma} e^{\imath \beta}\right)=\tilde{u}\left(r^{\gamma} e^{\imath(\beta-)}\right) \leqq 0 \quad(r \in J \cap(0, R)) .
$$

Thus, by (2.8) and (2.9), $H_{\theta}(-r) \leqq 0(r \in J \cap(0, R))$, i. e.

$$
h_{\theta}(-r)+\left(h_{1}\right)_{\theta}(-r) \leqq \cos \pi \alpha h_{\theta}(r)+A_{1} \quad(r \in J \cap(0, R)) .
$$

Combining (2.2), (2.3) and (2.10), we have

$$
A_{1}+r G_{1}^{\prime}(r) \geqq \alpha G_{1}(r) \quad(r \in J \cap(0, R)) .
$$

Here we note that

$$
\begin{aligned}
G_{1}(r)>C(\alpha) h_{\theta}(r)>\frac{C(\alpha)}{\pi} \int_{0}^{\pi} h_{\theta}\left(r e^{i \theta}\right) d \theta=\frac{C(\alpha)}{\pi} h(-r) \\
=\frac{C(\alpha)}{\pi} B(r)=\frac{C(\alpha)}{\pi} T\left(r^{r}\right) \quad(r \in(0, R)),
\end{aligned}
$$

by (2.2) and (1.2). Therefore by (2.11) and (2.12)

$$
r G_{1}^{\prime}(r)>(\alpha-\varepsilon) G_{1}(r) \quad\left(r \in J \cap\left(r_{3}, R / 2\right), r_{3}=r_{3}(\varepsilon)>0\right) .
$$

Since $G_{1}^{\prime}(r)>0$ a. e. in $0<r \leqq R / 2$, we deduce from (2.6) and (2.13) that

$$
\int_{J \cap\left(r_{4}, R / 2\right)} \frac{d t}{t}<\frac{1}{\alpha-\varepsilon}\left(C_{1}+\log T\left(R^{r} e^{r}\right)\right)
$$


where $r_{4}=\max \left(r_{2}, r_{3}\right)$.

Now, we choose $R=R_{n} \uparrow \infty$ such that

$$
C_{1}+\log T\left(R^{r} e^{r}\right)<(\mu+\varepsilon) \log \left(\frac{R}{2}\right)^{r} \quad(\varepsilon>0)
$$

Then

$$
\frac{1}{\log (R / 2)} \int_{J \cap\left(r_{4},(R / 2)\right)} \frac{d t}{t}<\frac{\gamma(\mu+\varepsilon)}{\alpha-\varepsilon},
$$

so that

$$
\frac{1}{\log (R / 2)^{\gamma}} \int_{I \cap\left(r_{4} \gamma,(R / 2) \gamma\right)} \frac{d t}{t}<\frac{\gamma(\mu+\varepsilon)}{\alpha-\varepsilon} .
$$

Hence

$$
\underline{\log \operatorname{dens}} I \leqq \frac{\gamma(\mu+\varepsilon)}{\alpha-\varepsilon},
$$

and so, since we may choose $\varepsilon$ arbitrarily small.

$$
\underline{\log \operatorname{dens}} I \leqq \frac{\gamma \mu}{\alpha}=\frac{\mu}{\lambda} .
$$

This completes the proof of Theorem 2 .

\section{Proof of Theorem 4 .}

Define $\beta, \gamma$ and $\alpha$ as in $\S 1$. Put $B(t)=T\left(t^{\gamma}\right)(0<t<\infty)$. Since $B(t)=O\left(\left(t^{r}\right)^{\rho+\varepsilon}\right)$ $\leqq O\left(t^{\alpha}\right)(t \rightarrow \infty)$, the Poisson integral

$$
h(z)=\frac{1}{\pi} \int_{0}^{\infty} \frac{r \sin \theta}{t^{2}+r^{2}+2 t r \cos \theta} B(t) d t \quad\left(z=r e^{i \theta}\right)
$$

is harmonic in the slit plane $|\arg z|<\pi$, is zero on the positive axis and tends to $B(r)$ as $\theta \rightarrow \pi-$. Also, (1.2) and (1.3) hold.

Nonconstancy of $u(z)$ implies the existence of a number $r_{2}>0$ such that $B_{1}\left(r_{2}\right)>0$. Hence

$$
\begin{aligned}
\pi h_{\theta}\left(r_{2}\right) & =\int_{0}^{\infty} \log \left(1+\frac{r_{2}}{t}\right) d B_{1}(t) \geqq \int_{0}^{r_{2}} \log \left(1+\frac{r_{2}}{t}\right) d B_{1}(t) \\
& =B_{1}\left(r_{2}\right) \log 2+\int_{0}^{r_{2}} B_{1}(t) \frac{r_{2}}{r_{2}+t} \frac{d t}{t} \geqq B_{1}\left(r_{2}\right) \log 2>0 .
\end{aligned}
$$

By an inequality of Denjoy [6],

$$
\int_{r}^{\infty}\left\{\log \left|1-\frac{t}{s}\right|-\cos \pi \alpha \log \left(1+\frac{t}{s}\right)\right\} \frac{d t}{t^{1+\alpha}}>C(\alpha) \frac{\log (1+r / s)}{r^{\alpha}}
$$

for all $r>0$, where $C(\alpha)=(1-\cos \pi \alpha) / \alpha$. We take the integral $d B_{1}(s)$ of each term in (3.2). Then, using the Fubini's theorem, we have 


$$
\int_{r}^{\infty}\left\{h_{\theta}(-t)-\cos \pi \alpha h_{\theta}(t)\right\} \frac{d t}{t^{1+\alpha}}>C(\alpha) \frac{h_{\theta}(r)}{r^{\alpha}} .
$$

On integration by parts this becomes

$$
\int_{r}^{\infty} F(t) \frac{d t}{t^{1+\alpha}}>0
$$

where $F(t)=h_{\theta}(-t)-h_{\theta}(t)+C(\alpha) t h_{\theta}^{\prime}(t)$. We define

$$
G_{1}(r)=r^{\alpha} \int_{r}^{\infty} F(t) \frac{d t}{t^{1+\alpha}}+C(\alpha) h_{\theta}(r) \quad(r>0) .
$$

Then

$$
r G_{1}^{\prime}(r)=\alpha r^{\alpha} \int_{r}^{\infty} F(t) \frac{d t}{t^{1+\alpha}}+h_{\theta}(r)-h_{\theta}(-r) \quad \text { a. e. . }
$$

Since $G_{1}(r)$ is absolutely continuous, this implies that $G_{1}(r)$ is monotonic increasing, and so $\log G_{1}(r)$ is absolutely continuous. Therefore

$$
\int_{r_{2}}^{r} \frac{G_{1}^{\prime}(t)}{G_{1}(t)} d t=\log G_{1}(r)-\log G_{1}\left(r_{2}\right) .
$$

Now, by (11) and (12) in [1],

$$
\begin{aligned}
\pi h_{\theta}(s) & =\int_{0}^{\infty} \log \left(1+\frac{s}{t}\right) d B_{1}(t) \\
& =\int_{0}^{\infty} \frac{s}{t+s} d B(t) \\
& =\int_{0}^{\infty} \frac{s}{(t+s)^{2}} B(t) d t=O\left(s^{\gamma \rho+\varepsilon}\right) \quad(s \longrightarrow \infty)
\end{aligned}
$$

for every $\varepsilon>0$, and therefore

$$
\begin{aligned}
G_{1}(r) & =r^{\alpha} \int_{r}^{\infty}\left\{h_{\theta}(-t)-\cos \pi \alpha h_{\theta}(t)\right\} \frac{d t}{t^{1+\alpha}} \\
& \leqq r^{\alpha}(1-\cos \pi \alpha) \int_{r}^{\infty} h_{\theta}(t) t^{-1-\alpha} d t=O\left(r^{\gamma \rho+\varepsilon}\right) \quad(r \longrightarrow \infty) .
\end{aligned}
$$

Combining (3.1), (3.4) and (3.5), we have

$$
\int_{r_{2}}^{r} \frac{G_{1}^{\prime}(t)}{G_{1}(t)} d t<(\gamma \rho+o(1)) \log r \quad(r \longrightarrow \infty) .
$$

Next, consider the harmonic function $H(z)$ in the upper half plane defined by

$$
H\left(r e^{i \theta}\right)=h\left(r e^{i \theta}\right)+\cos \pi \alpha h\left(r e^{\imath(\pi-\theta)}\right)+A_{1}(\pi-\theta),
$$

where $A_{1}$ is a large positive constant determined later. The boundary values of $H$ satisfy 


$$
\left\{\begin{array}{l}
H(-r)=T\left(r^{\gamma}\right) \quad(0 \leqq r<\infty), \\
H(r)=\cos \pi \alpha T\left(r^{r}\right)+A_{1} \pi \quad(0<r<\infty) .
\end{array}\right.
$$

Set

$$
v(z)=u^{\#}\left(z^{\gamma}\right)
$$

Then

$$
\left\{\begin{array}{l}
v(-r)=u^{\#}\left(r^{\gamma} e^{\imath \beta}\right) \leqq T\left(r^{\gamma}\right)=H(-r) \quad(0 \leqq r<\infty), \\
\left.v(r)=u^{\#}\left(r^{\gamma}\right)=N\left(r^{\gamma}\right), u_{2}\right) \leqq(1-\delta) T\left(r^{\gamma}\right)+C \\
\left(r \geqq r_{1}=r_{0}^{1 / r}, \quad C: \text { a positive constant }\right) .
\end{array}\right.
$$

Hence, if we choose $A_{1} \pi=\max \left\{C, N\left(r_{0}, u_{2}\right)\right\}$, then

$$
v(z) \leqq H(z)
$$

holds on the real axis. Since $v(z)$ and $H(z)$ are both $O\left(r^{\alpha}\right)$ in the upper half plane as $r \rightarrow \infty$, and since $\alpha \leqq 1 / 2$, we conclude that

in the upper half plane.

$$
v(z) \leqq H(z)
$$

Now, let $I$ and $J$ be as in $\S 2$. Then the same reasoning as in $\S 2$ gives

$$
H_{\theta}(-r) \leqq 0 \quad(r \in J) .
$$

In view of the definition of $H$, this can be written

$$
h_{\theta}(-r)-\cos \pi \alpha h_{\theta}(r) \leqq A_{1} \quad(r \in J) .
$$

Hence

$$
A_{1}+r G_{1}^{\prime}(r) \geqq \alpha G_{1}(r) \quad(r \in J) .
$$

Since

$$
G_{1}(r)>C(\alpha) h_{\theta}(r)=\frac{C(\alpha)}{\pi} \int_{0}^{\infty} \frac{r}{(t+r)^{2}} B(t) d t \geqq \frac{C(\alpha) B(r)}{\pi} \int_{r}^{\infty} \frac{r}{(t+r)^{2}} d t=\frac{C(\alpha) B(r)}{2 \pi},
$$

we have

$$
r G_{1}^{\prime}(r) \geqq(\alpha-\varepsilon) G_{1}(r) \quad\left(r \in J \cap\left(r_{3}, \infty\right), r_{3}=r_{3}(\varepsilon)>0\right) .
$$

Combining (3.6) with (3.7), we have

$$
\int_{J \cap\left(r_{4}, r\right)} \frac{d t}{t}<\frac{\gamma \rho+o(1)}{\alpha-\varepsilon} \log r \quad(r \rightarrow \infty),
$$

where $r_{4}=\max \left(r_{2}, r_{3}\right)$, and arguing as from (2.14), we deduce that

$$
\overline{\log \operatorname{dens}} I \leqq \frac{\gamma \rho}{\alpha}=\frac{\rho}{\lambda} \text {. }
$$

This completes the proof of Theorem 4 . 


\section{REFERENCES}

[1] A. Baernstein II; A generalization of the $\cos \pi \rho$ theorem, Trans. Amer. Math. Soc. 193 (1974), 181-197.

[2] A. BAERnStein II; Integral means, univalent functions and circular symmetrization, Acta Math. 133 (1975), 139-169.

[3] A. BAERnstein II; Regularity theorems associated with the spread relation, J. d'Analyse Math. 31 (1977), 76-111.

[4] P.D. BARRY; On a theorem of Besicovitch, Quart. J. Math. Oxford (2) 14 (1963), 293-302.

[5] P.D. BARrY; On a theorem of Kjellberg, Quart. J. Math. Oxford (2) 15 (1964), 179-191.

[6] A. Denjoy; Sur un théorème de Wiman, C.R. Acad. Sc1. Parıs 193 (1931), 828 -830 .

[7] B. KJEllberg; On the minimum modulus of entire functions of lower order less than one, Math. Scand. 8 (1960), 189-197.

Department of Mathematics,

Daido Institute of Technology,

Daido-cho, Minami-Ku, Magoya, Japan 